\title{
Optimized matching modes of bioelectrochemical module and anaerobic sludge in the integrated system for azo dye treatment
}

\author{
Fanying Kong a , Aijie Wang ${ }^{\mathrm{a}, \mathrm{b}, *}$, Hong-Yu Ren ${ }^{\mathrm{a}}$ \\ ${ }^{a}$ State Key Laboratory of Urban Water Resource and Environment, School of Municipal and Environmental Engineering, Harbin Institute of Technology, Harbin 150090, China \\ ${ }^{\mathrm{b}}$ Research Center for Eco-Environmental Sciences, Chinese Academy of Sciences, Beijing 100085, China
}

\section{H I G H L I G H T S}

- Matching modes of BES and anaerobic sludge in integrated system were optimized.

- $1 / 4$ cathode soaking in the sludge with spiral distributor in down-flow was the best.

- Current, EIS and pH clearly demonstrated the efficient electron/proton transfer.

- Down-flow mode favored the efficient use of cathode and improved decolorization.

- Spiral distributor may create a spiral flow channel across cathode-microb es-solution.

\section{A R T I C L E I N F O}

\section{Article history:}

Received 6 May 2015

Received in revised form 29 May 2015

Accepted 1 June 2015

Available online 4 June 2015

\section{Keywords:}

Integrated system

Bioelectrochemical system (BES)

Anaerobic sludge reactor (ASR)

Matching mode

Decolorization

\section{G R A P H I C A L A B S T R A C T}

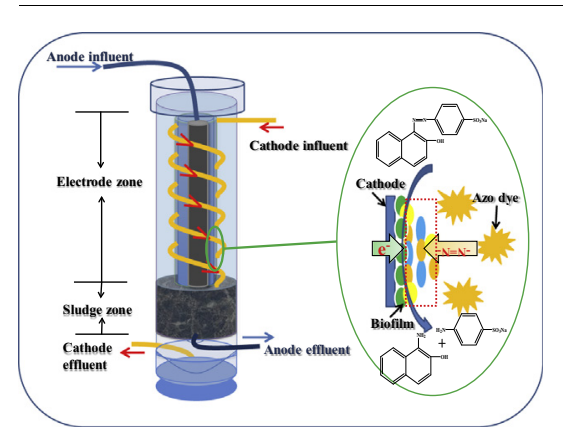

\begin{abstract}
A B S T R A C T
In this work, three matching modes (relative positions, catholyte flow sequences, and flow regimes) of bioelectrochemical module and anaerobic sludge were evaluated and optimized for azo dye treatment in the integrated system with embedding modular bioelectrochemical system into anaerobic sludge reactor. Results showed that it was favorable to operate this integrated system under the condition of $1 / 4$ cathode soaking into sludge with spiral distributor in down-flow direction. Current, electrochemical impedance spectroscopy and $\mathrm{pH}$ clearly demonstrated the important role of $1 / 4$ soaking in electron/ proton transfer. The down-flow direction flowed through electrode zone and then sludge zone could benefit to the efficient use of cathode and improve AO7 treatment. Furthermore, the positive effect of spiral catholyte distributor might be due to its promoting role in mixing and creating a spiral flow channel around the cathode electrode-microbes-solution interface. These results exhibited great potential for matching modular bioelectrochemical system with anaerobic treatment process.
\end{abstract}

(c) 2015 Elsevier Ltd. All rights reserved.

\section{Introduction}

For refractory wastewater treatment, using an appropriate pretreatment technology to improve its biodegradability has been

\footnotetext{
* Corresponding author at: State Key Laboratory of Urban Water Resource and Environment, School of Municipal and Environmental Engineering, Harbin Institute of Technology, Harbin 150090, China. Tel./fax: +86 45186282195.

E-mail addresses: fanyingkong@163.com(F. Kong),waj0578@163.com(A.Wang), rhongyu@126.com (H.-Y. Ren).
}

recognized as an effective treatment model before inducing into the conventional biological treatment process (Sun et al., 2011). The bioelectrochemical system (BES) as a novel and promising approach is designed and constructed to utilize bioelectrochemical or oxidization-redox processes to remove pollutants from contaminated water within a more controlled environment (electricity generation or a low applied voltage to reach the redox potential) (Kong et al., 2014b,c; Li et al., 2014; Ruiz et al., 2015). It is very suitable for treating wastewater rich in unsaturated bonds such as azo 
dye and metal (Huang et al., 2014; Mu et al., 2009; Sun et al., 2011; Tao et al., 2014; Wang and Ren, 2014), being established world widely as an alternative pretreatment system.

Further considering the conjunction of BES with current technologies, there has been growing interest in combining anaerobic sludge reactor with BES for its wide use in wastewater treatment (Li et al., 2014; Solís et al., 2012). It would be a great potential application for industry feasibility, especially with BES module embedding into the traditional anaerobic sludge reactor. It has been found that the performance of azo dye wastewater treatment can be significantly improved by embedding the BES module with surrounding electrode deployment into anaerobic sludge reactor (ASR) to form integrated BES-ASR in the treatment process (Kong et al., 2015). This integrated process not only allowed electrons produced from wastewater at anode to be an electron donor for removing pollution at cathode, but also realized some organics removal through the anaerobic process of the sludge, implying good synergy between these two technologies. Although the feasibility of the integrated system with BES and ASR has been demonstrated (Kong et al., 2015), however, the knowledge on the technology combination for the performance promotion of wastewater treatment is still not sufficient. As a newly designed reactor, the matching modes of BES module and anaerobic sludge are needed to be optimized to determine the optimal parameters and operation conditions in order to improve its overall performance.

In this study, the matching modes of the integrated system, including relative positions of cathode electrode and sludge, catholyte flow sequences through electrode zone and sludge zone, and flow regimes between cathode and sludge, were investigated aiming to provide a theoretical basis and reference for future practical application.

\section{Methods}

\subsection{Configuration of the integrated system}

The BES-ASR integrated reactor was developed by inserting sleeve-type BES module (inner tube full of holes: ID $3 \mathrm{~cm} \times \mathrm{H}$ $10 \mathrm{~cm}$ ) into an anaerobic sludge reactor (ASR: outer tube ID $8 \mathrm{~cm} \times \mathrm{H} 10 \mathrm{~cm}$ ), as previously described with the anaerobic sludge at the bottom (Kong et al., 2015). Carbon brush anode was placed in the center of the inner tube, surrounding the carbon brush cathode in the outer tube with the electrode ratio of 1:3. The distance between the anode and the cathode was $1 \mathrm{~cm}$. The working volume for the inner anode chamber was $70 \mathrm{~mL}$, resulting in the working volume for cathode to be $210 \mathrm{~mL}$ as the inner tube inserted, with the anode and cathode volume ratio of $1: 3$, too. The anode, cathode and the reference electrode were connected to a data acquisition (Keithley 2700, Keithley, Co., Ltd., USA) with external resistance of $20 \Omega$.

\subsection{Experimental operation}

The anolyte was $1 \mathrm{~g} \mathrm{~L}^{-1} \mathrm{NaAc}$ with nutrient medium as described previously (Kong et al., 2015), and the catholyte was acid orange 7 (AO7, skeletal formula can be seen in the Supporting information Fig. S1) with the same nutrient medium. Since the anode potential stabilized at about $-0.4 \mathrm{~V}$ with $\mathrm{NaAc}$ as the electron donor, the integrated system was applied voltage of $0.5 \mathrm{~V}$ to reach the cathode potential of $-0.9 \mathrm{~V}$, which was the AO7 redox potential (Kong et al., 2013).

Experiments were performed to optimize the treatment performance in the integrated system with three different matching modes. (1) The relative positions of cathode electrode and sludge. Four kinds of relative positions between cathode electrode and sludge were considered with different soaking proportion of cathode into sludge, including 0 soaking (placing all of the cathode carbon brush in the supernatant fluid above the sludge zone), 1/4 soaking (placing quarter of the cathode carbon brush into the sludge), $1 / 2$ soaking (placing half of the cathode carbon brush into the sludge with the other half into the supernatant fluid), and 1 soaking (placing all the cathode carbon brush into the sludge). The reactors were start-up at a concentration of $100 \mathrm{mg} \mathrm{L}^{-1}$ at HRT $=12 \mathrm{~h}$. When the biofilm adapt the AO7 toxicity, the AO7 concentration was gradually increased up to the concentration of $300 \mathrm{mg} \mathrm{L}^{-1}$. Each stage was operated no less than 20 days. The stable data of the operation was used to compare the effect of relative positions on the AO7 decolorization. (2) The catholyte flow sequences through electrode zone and sludge zone. The cathode electrode was placed in the water zone above the anaerobic sludge bed of the sludge zone, to become the electrode zone. The catholyte flow sequences were expressed by the flow directions, including the up-flow direction and down-flow direction. In the up-flow direction, the azo dye was firstly flowed through the sludge and then the cathode electrode; conversely, it would be firstly through the cathode electrode and then the anaerobic sludge in down-flow direction. In this part, it was focused on the influence of the catholyte flow sequence through cathode electrode and sludge with HRT of $10 \mathrm{~h}$ in the $1 / 4$ soaking integrated reactor. (3) The flow regimes between cathode and sludge. Two different shapes of catholyte distributor were compared between planar distributor and spiral distributor to investigate the flow regimes. The planar distributor, a porous Perspex plate $(2 \mathrm{~mm}$ thick with a pore size of $2 \mathrm{~mm}$ and a fractional perforated area of $20 \%$ ) was placed on the top of cathode electrode zone with down-flow direction to form vertical flow. The spiral distributor, a spiral tube (inner diameter of $1 \mathrm{~cm}, 2 \mathrm{~mm}$ thick with a pore size of $2 \mathrm{~mm}$ and a fractional perforated area of 20\%) was surrounding the cathode electrode to form spiral flow. The HRT $=8 \mathrm{~h}$.

\subsection{Analysis and calculation}

AO7 concentration was measured at $\lambda_{\max }=484 \mathrm{~nm}$ by UV-vis scanning spectrophotometer (Shimadzu UV2550, Japan). AO7 decolorization and chemical oxygen demand (COD) were identified and calculated according to Fernando et al. (2012). Anode and cathode as well as the reference electrode were connected to the data acquisition unit to record the anode potential, cathode potential, and current every $10 \mathrm{~min}$. Electrochemical impedance spectroscopy (EIS) measurements were carried at open circuit condition in a frequency range of $0.01-10^{5} \mathrm{~Hz}$ with a perturbation signal of $10 \mathrm{mV}$ using electrochemical workstation (model-660D, CH Instruments Inc., USA) (Kong et al., 2014a).

\section{Results and discussion}

\subsection{Optimization of the relative positions between cathode electrode and sludge}

\subsubsection{Azo dye decolorization with different relative positions}

The BES-ASR integrated reactors with 0 soaking, 1/4 soaking, $1 / 2$ soaking, and 1 soaking were applied to investigate the effect of relative positions between cathode electrode and sludge on decolorization performance.

Results showed that the relative positions of cathode and sludge in the integrated system significantly affected AO7 decolorization efficiency when the initial concentration gradually increased from 100 to $300 \mathrm{mg} \mathrm{L}^{-1}$ at $\mathrm{HRT}=12 \mathrm{~h}$. At $100 \mathrm{mg} \mathrm{L}^{-1}$, the decolorization efficiency was $87.6 \pm 2.9 \%, 98.6 \pm 2.0 \%, 85.1 \pm 2.2 \%$, and $69.8 \pm 3.7 \%$ for 0 soaking, $1 / 4$ soaking, $1 / 2$ soaking, and 1 soaking, respectively. 
As it increased to $300 \mathrm{mg} \mathrm{L}^{-1}$, the corresponding decolorization efficiency was $61.2 \pm 3.4 \%, 80.9 \pm 1.9 \%, 57.3 \pm 4.1 \%$, and $37.8 \pm 5.1 \%$, respectively. The comparison results showed that the $1 / 4$ soaking performed the best decolorization performance, which was about $10 \%$ higher than 0 soaking and $1 / 2$ soaking, and $40 \%$ higher than 1 soaking (Fig. 1A). The $1 / 4$ soaking exhibited no apparent inhibitory effect of AO7 and exposed more advantages especially in higher concentration, followed by 0 soaking, $1 / 2$ soaking, and 1 soaking. Even at the concentration up to $300 \mathrm{mg} \mathrm{L}^{-1}$, the decolorization rate of $1 / 4$ soaking was increased to $20.3 \pm 1.0 \% \mathrm{mg} \mathrm{L}^{-1} \mathrm{~h}^{-1}$ (Fig. $1 \mathrm{~B}$ ). However, the decolorization rates for 0 soaking, $1 / 2$ soaking and 1 soaking stayed stable at $13.8 \pm 0.8 \%, 13.3 \pm 1.1 \%$, and $8.6 \pm 0.7 \%$, respectively, or even decreased as concentration increased from 200 to $300 \mathrm{mg} \mathrm{L}^{-1}$ (Fig. 1B). As can be seen, the $1 / 4$ cathode soaking into sludge could improve the decolorization performance, but the other soaking ways ( $1 / 2$ soaking and 1 soaking) were not conducive to promote decolorization and were even inferior to 0 soaking. The reason might be that in the $1 / 4$ soaking reactor, the function microorganism in the sludge could easily migrate to the upper part of the cathode electrode through the partial soaking electrode in the process of continuous operation. It would be easier to form biofilms at cathode or to be the suspended microbes in solution. In addition, it might be relative to the proton transfer through the membrane and/or electron transfer through the electrode. If the electrode was soaking too much, especially for 1 soaking, the biofilm covered on the membrane would become proton transfer resistance, and biofilm that not be bio-electrocatalytically active around the cathode may result in electron transfer resistance. It may also develop large amounts of biomass that can act to restrict mass transfer process (Michie et al., 2014).
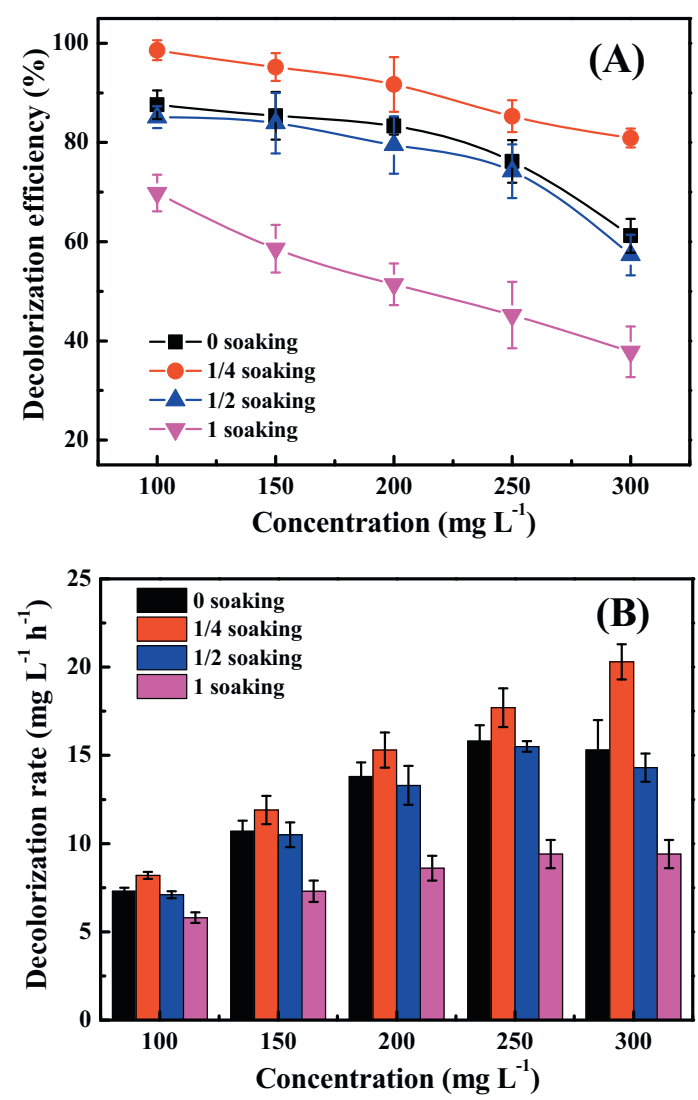

Fig. 1. Effect of relative positions in the integrated system on acid orange 7 decolorization efficiency (A) and decolorization rate (B) with different soaking proportion of cathode into sludge at different concentrations, including 0 soaking, $1 / 4$ soaking, $1 / 2$ soaking, and 1 soaking (HRT = $12 \mathrm{~h}$ ).

\subsubsection{The $\mathrm{pH}$ and electrochemical characterizations with different relative positions}

To discover the reason for improved decolorization with $1 / 4$ soaking and inhibitive performance with 1 soaking, the $\mathrm{pH}$ and the electrochemical characterizations (electrode potential, current, and EIS) were studied to evaluate the proton transfer and electron transfer compared with other relative positions.

The $1 / 4$ soaking and 0 soaking eliminated the membrane $\mathrm{pH}$ gradient as the $\mathrm{pH}$ of anolyte/catholyte was nearly neutral (Fig. 2), indicating the efficient proton transfer from anode chamber to cathode chamber through the membrane (Ruiz et al., 2015). However, for $1 / 2$ soaking, $\mathrm{pH}$ of anolyte decreased to $6.59 \pm 0.03$ and $\mathrm{pH}$ of catholyte increased to $7.28 \pm 0.11$. Especially for 1 soaking, the $\mathrm{pH}$ reached $6.29 \pm 0.11$ and $7.66 \pm 0.17$, respectively (Fig. 2). According to the fact that the proton in the solution is directly involved in the oxidation/reduction reaction at anode/cathode (Ruiz et al., 2015), the decreased pH of anolyte and increased $\mathrm{pH}$ of catholyte exhibited the proton transfer limitation through the membrane in the integrated system when the cathode was soaking into sludge with a much larger proportion (1/2 soaking and 1 soaking), which would result in the lower decolorization (Fig. 1).

Meanwhile, thick biofilm across or on the electrode in the soaking cathode could limit electron transfer from cathode to azo dye, which can be obtained from the current data (Fig. 3B). The current began to rise as the catholyte (100 $\mathrm{mg} \mathrm{L}^{-1} \mathrm{AO}$ ) was continuously flowed into the cathode chamber, indicating the successfully electrochemical reduction reaction of AO7. After about $360 \mathrm{~h}$, the current of each reactor was gradually stabilized at $0.013 \pm 0.002$, $0.018 \pm 0.005,0.016 \pm 0.002$, and $0.013 \pm 0.002 \mathrm{~A}$, respectively for 0 soaking, $1 / 4$ soaking, $1 / 2$ soaking, and 1 soaking (Fig. $3 B$ ), indicating the formation of cathode electrochemical active biofilm in the integrated system. As the $\mathrm{AO} 7$ concentration subsequently increased to $300 \mathrm{mg} \mathrm{L}^{-1}$, the current significantly increased to $0.029 \pm 0.004$ A (0 soaking), $0.047 \pm 0.003$ A (1/4 soaking), $0.034 \pm 0.002 \mathrm{~A}(1 / 2$ soaking), and $0.023 \pm 0.002 \mathrm{~A}$ (1 soaking) (Fig. 3B), which was $223 \%, 261 \%, 213 \%$, and $177 \%$ higher compared to that at $100 \mathrm{mg} \mathrm{L}^{-1}$. It suggested that increasing the concentration of azo dye to $300 \mathrm{mg} \mathrm{L}^{-1}$ had no obvious inhibition to the cathode electrochemical microorganisms. According to that a higher current may be attributed to a more efficient electron transfer or a lower cathode resistance (Kong et al., 2015), the increased concentration probably promoted the electrochemical reaction rate at cathode, resulting in the higher current, which was consistent with decolorization rate in Fig. 1. More importantly, the cathode soaking into sludge might have both positive and negative effects,

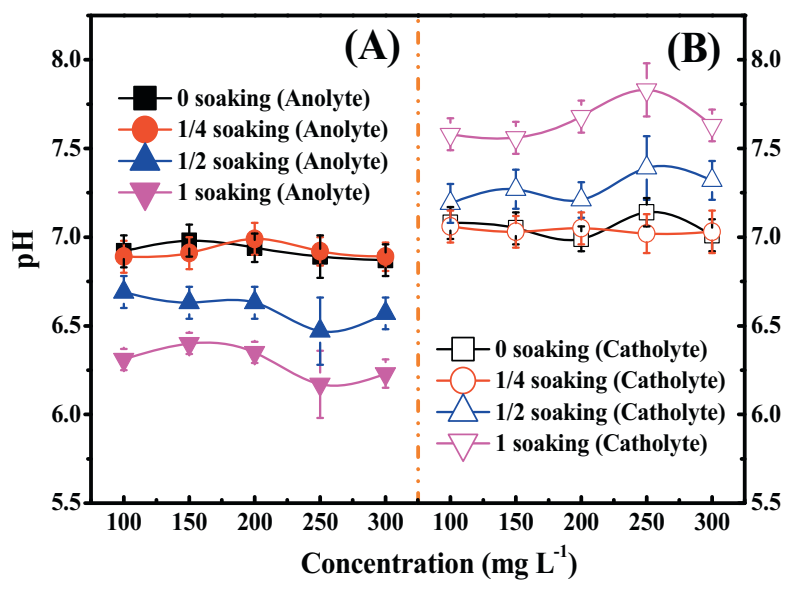

Fig. 2. Effect of relative positions on the $\mathrm{pH}$ of anolyte (A) and catholyte (B) with different soaking proportion of cathode into sludge at different concentrations, including 0 soaking, $1 / 4$ soaking, $1 / 2$ soaking, and 1 soaking (HRT = $12 \mathrm{~h}$ ). 

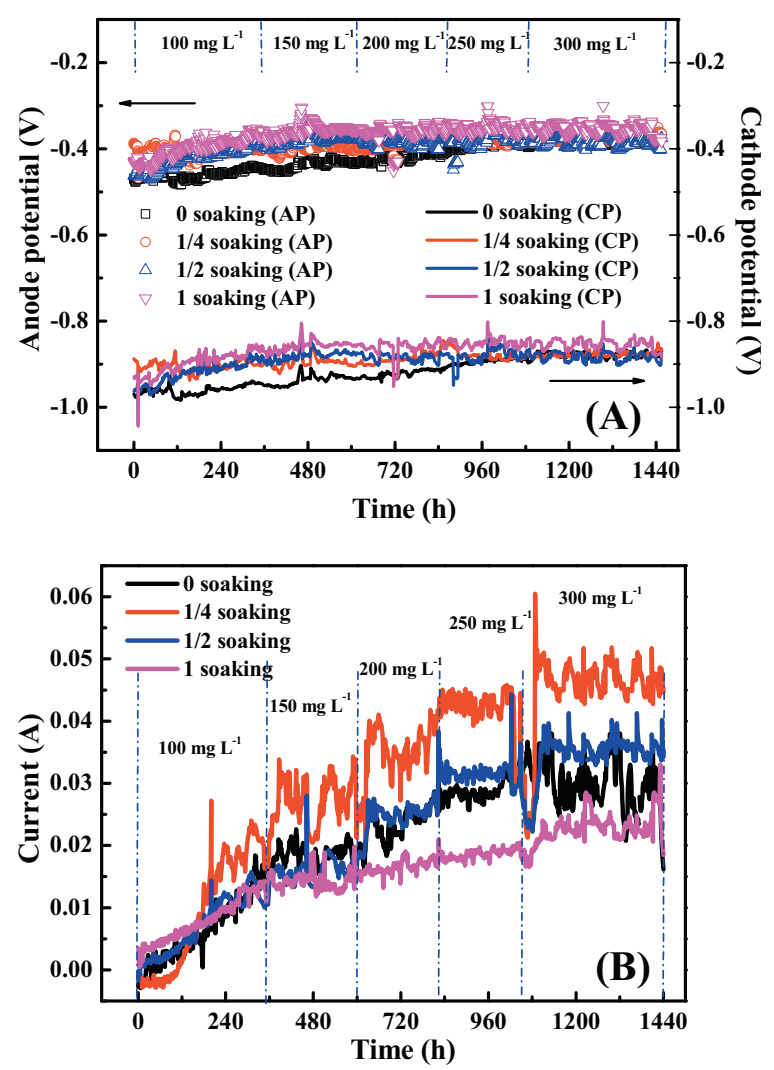

Fig. 3. Effect of relative positions on the electrode potential (A) and current (B) with different soaking proportion of cathode into sludge at different concentrations, including 0 soaking, $1 / 4$ soaking, $1 / 2$ soaking, and 1 soaking $(H R T=12 \mathrm{~h})$.

depending on the soaking proportion. The current of $1 / 4$ soaking $(0.047 \pm 0.003 \mathrm{~A})$ at $300 \mathrm{mg} \mathrm{L}^{-1}$ was 1.6 -fold higher than 0 soaking $(0.029 \pm 0.004 \mathrm{~A}), 1.4$-fold higher than $1 / 2$ soaking $(0.034 \pm 0.002$ A) and 2.0-fold higher than 1 soaking $(0.023 \pm 0.002 \mathrm{~A})$ (Fig. $3 \mathrm{~B})$. It can be seen that the $1 / 4$ soaking exhibited the highest current, likely due to that the $1 / 4$ soaking might have relative high microbial activity, leading to a change of both microbial community structure and diversity (Pant et al., 2010). Although sludge could benefit the microorganism attachment and cause higher microbial biomass yield, electron transfer to the azo dye and mass transfer to the biofilm could be constricted when an electrogenic biofilm became thicker in the 1 soaking system. This would result in the current limited not only by biocatalysis of the azo dye, but also by diffusion of catholyte to the biofilm surface (Michie et al., 2014). Thus, bioelectrochemical reaction might contribute less to the azo dye decolorization in 1 soaking reactor in comparison with other relative positions and most of the $\mathrm{AO} 7$ might be removed by anaerobic biological process.

It should be noted that different from the current results, anode potentials with different relative positions were stable at about $-0.41 \pm 0.05 \mathrm{~V}$ when AO7 concentration increased from 100 to $300 \mathrm{mg} \mathrm{L}^{-1}$. It might be due to the dual chamber configuration that separated anode from cathode, which cause no toxic transfer of AO7 to the anode and no microbial activity inhibition at anode. As $0.5 \mathrm{~V}$ voltage applied, the cathode potentials were stable at $-0.91 \pm 0.05 \mathrm{~V}$ (Fig. $3 \mathrm{~A}$ ), reaching the AO7 redox potential. If further considering the practical application, a constant potential $-0.9 \mathrm{~V}$ can be applied to cathode to achieve effective reduction of azo dye in the case of low-cost integrated system operation.

The EIS were also obtained to determine the detailed information on charge transfer and mass transfer in the integrated system with different relative positions of cathode and sludge.
Nyquist curves of the integrated reactors were typical of the semicircle high frequency region and ray low frequency region (Fig. 4A). Results illustrated that the relative positions of cathode and sludge played an important role in internal resistances. The $1 / 4$ soaking reactor performed the lowest internal resistance of $3.8 \Omega$, lower than 0 soaking reactor $(5.8 \Omega), 1 / 2$ soaking reactor $(35.1 \Omega$ ) and 1 soaking reactor $(242.0 \Omega$ ) (Fig. 4A). The order was consistent with the decolorization and current results (Figs. 1 and 3B), indicating that the performance of integrated system was probably attributed to the resistance of reactor, which was composed of ohmic resistance $\left(R_{\mathrm{ohm}}\right)$, charge transfer resistance $\left(R_{\mathrm{ct}}\right)$ and diffusion resistance $\left(R_{\mathrm{diff}}\right)$. The proportion of $R_{\mathrm{ct}}$ in the internal resistance accounted for $51.7 \%, 73.7 \%, 83.8 \%$, and $83.5 \%$ with 0 soaking, $1 / 4$ soaking, $1 / 2$ soaking and 1 soaking, respectively (Fig. $4 \mathrm{~A}$ and Table 1 ). It indicated that the internal resistances were probably derived from the $R_{\mathrm{ct}}$, demonstrating the different decolorization performance with different relative positions of cathode and sludge significantly influenced by the $R_{\mathrm{ct}}$. Results showed that the $R_{\mathrm{ct}}$ of 1 soaking $(202.1 \Omega)$ were about 70 -fold higher than the 0 soaking $(3.0 \Omega$ ) and $1 / 4$ soaking $(2.8 \Omega$ ), and 7 -fold higher than $1 / 2$ soaking $\left(29.4 \Omega\right.$ ) (Fig. $4 \mathrm{~A}$ ). As $R_{\mathrm{ct}}$ was affected by the kinetics of the electrode reactions and was useful to evaluate the stability of electrode biofilm (He and Mansfeld, 2009), it indicated that when cathode was placed in the aqueous phase ( 0 soaking) or $1 / 4$ part in the sludge phase ( $1 / 4$ soaking), the electrochemical reaction would be accelerated for the efficient electron transfer. However, when cathode was placed in the sludge phase for 1 soaking, the electrode surface would attach microorganisms and form a thick biofilm, reducing the efficient contact area of the electrode with azo dye, and then resulting in the slow reaction rate (small current) of electrochemical reaction. This also can be further demonstrated from the Bode curves (Fig. 4B). The
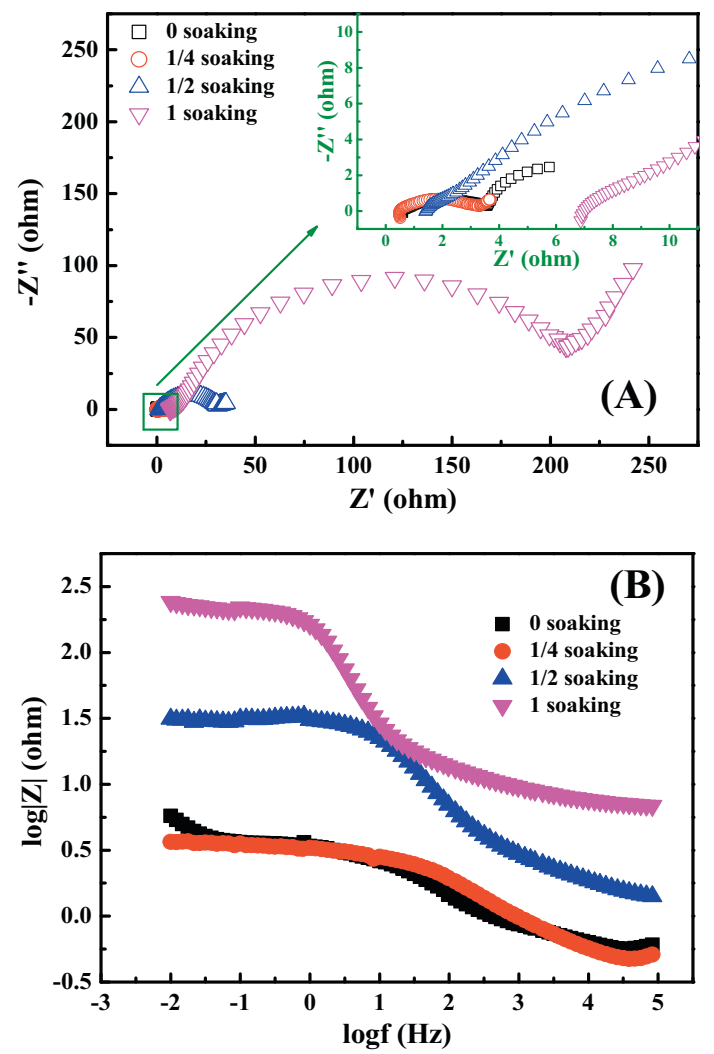

Fig. 4. EIS for integrated bioelectrochemical system-anaerobic sludge reactor with different relative positions of cathode and sludge, including the Nyquist plot (A) and Bode plot (B). 
Table 1

Proportion of resistance in the integrated system with different relative positions.

\begin{tabular}{|c|c|c|c|c|c|c|c|}
\hline Relative positions & $R_{\mathrm{ohm}}(\Omega)$ & Proportion (\%) & $R_{\mathrm{ct}}(\Omega)$ & Proportion (\%) & $R_{\text {diff }}(\Omega)$ & Proportion (\%) & $R(\Omega)$ \\
\hline 0 soaking & 0.6 & 10.3 & 3.0 & 51.7 & 2.2 & 37.9 & 5.8 \\
\hline $1 / 4$ soaking & 0.5 & 13.2 & 2.8 & 73.7 & 0.5 & 13.1 & 3.8 \\
\hline $1 / 2$ soaking & 1.4 & 4.0 & 29.4 & 83.8 & 4.3 & 12.2 & 35.1 \\
\hline 1 soaking & 6.9 & 2.8 & 202.1 & 83.5 & 33.0 & 13.6 & 242.0 \\
\hline
\end{tabular}

Bode plots showed the impedance behavior of relative positions as a function of frequency. The low frequency region of the Bode curves represented the charge transfer resistance, which affected by the kinetics of the electrode reactions (Kong et al., 2013). Higher value in this region can be attributed to slower kinetics of charge transfer reactions associated with the redox process (He and Mansfeld, 2009), confirming that the AO7 reduced decolorization with 1 soaking exhibited higher impedance compared to other soaking positions. It also ascertained that the $1 / 4$ soaking was the best in $\mathrm{AO7}$ reduction rate at cathode, which was consisted with the decolorization results in Fig. 1.

It can be concluded that the cathode electrode in the sludge zone with $1 / 4$ soaking was more reasonable in the integrated system with embedding bioelectrochemical module into anaerobic sludge reactor. The current, EIS and $\mathrm{pH}$ clearly demonstrated the important role of $1 / 4$ soaking in electron transfer and proton transfer compared with 0 soaking, $1 / 2$ soaking and 1 soaking.

\subsection{Optimization of the catholyte flow sequences through electrode and sludge zone}

According to the principle of azo dye for reduction decolorization in sleeve-type BES (Kong et al., 2013), the electrode zone would be more effective than the anaerobic sludge zone owing to the sufficient electrons transferred from the anode oxidation to realize reduction decolorization of azo dye at cathode. Aiming to combine the synergy effect of the cathode with bioelectrochemical reaction and sludge with anaerobic reaction for decolorization, the catholyte flow sequences were compared between up-flow direction and down-flow direction in the integrated system.

Experiments were firstly performed in the $1 / 4$ soaking reactor under closed circuit and open circuit conditions to confirm the contribution of bioelectrochemical role in the integrated system, which was meaningful to the determine of flow sequence. Under the open circuit condition, the total decolorization efficiency was decreased from $21.4 \pm 1.8 \%$ to $14.8 \pm 1.4 \%$ when the concentration increased from 100 to $300 \mathrm{mg} \mathrm{L}^{-1}$ at HRT $=10 \mathrm{~h}$. While the decolorization efficiency through the sludge zone was from $19.3 \pm 1.9 \%$ to $13.2 \pm 1.6 \%$, which was similar to the total decolorization efficiency, exhibiting the scarcely decolorization (3\%) in the electrode zone (Fig. 5A). These results indicated that the presence of sludge zone played a major role in the decolorization with open circuit operation, probably due to the anaerobic microorganisms in the sludge that could achieve reduction decolorization with slow reaction (Solís et al., 2012). However, at higher concentrations, the influent load exceeded the processing capability of the anaerobic microorganisms, and the azo dye itself had a strong toxicity to them, finally leading to the decrease decolorization efficiency in the open circuit. Different from the open circuit condition, the total decolorization efficiency in the closed circuit was greatly up to $80 \%$ as AO7 concentration increased from 100 to $300 \mathrm{mg} \mathrm{L}^{-1}$ with a small voltage applied $(0.5 \mathrm{~V})$. The contribution to decolorization was the synergy effect of sludge zone $(20.6 \pm 0.5-17.5 \pm 1.8 \%)$ and electrode zone $(77.1 \pm 2.7-63.7 \pm 1.3 \%)$. It can be seen that the contribution of the sludge zone in the closed circuit was almost equivalent to the open circuit. The difference of the final treatment performance was mainly caused by the electrical stimulation
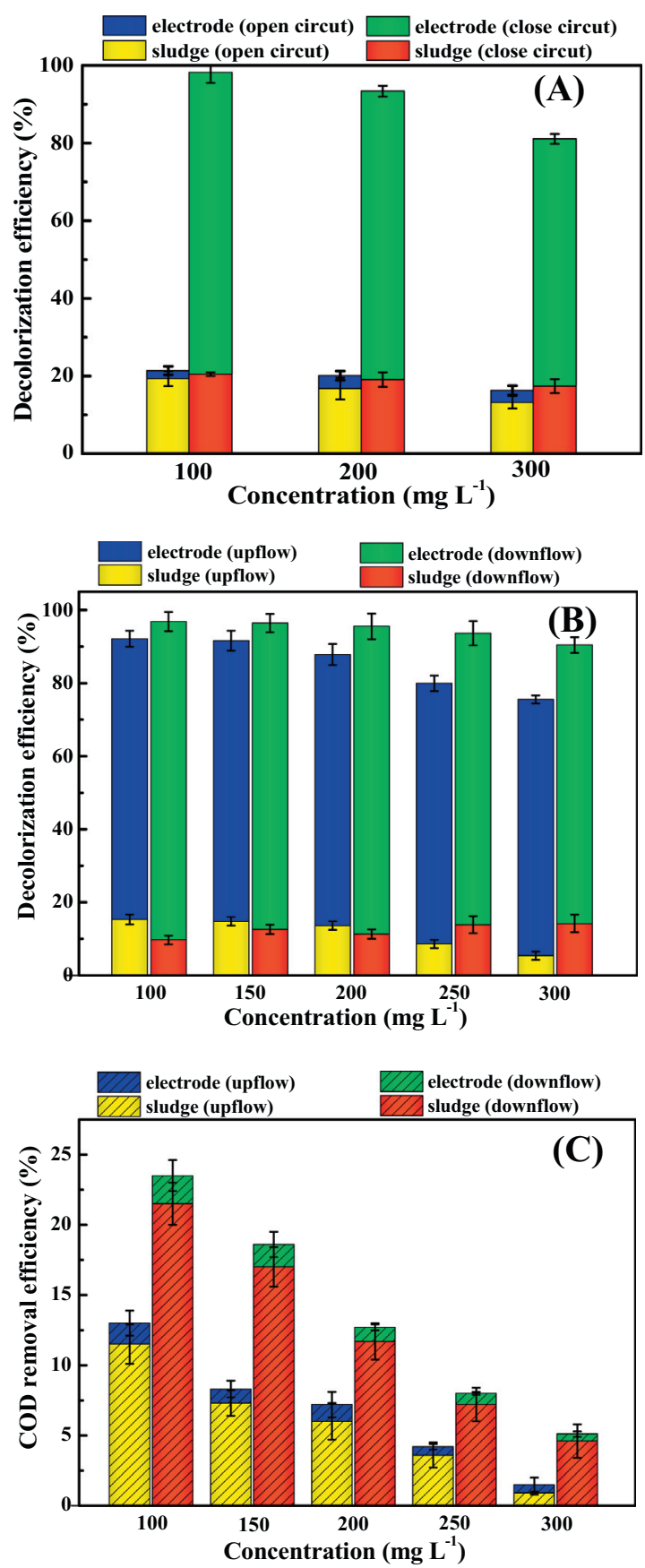

Fig. 5. The decolorization efficiency of sludge zone and electrode zone under open circuit and closed circuit conditions (A). The decolorization efficiency (B) and COD removal $(C)$ of sludge zone and electrode zone with different catholyte flow sequences (up-flow and down-flow) (HRT = $10 \mathrm{~h}$ ).

electrodes in the electrode zone. These results testified that in the integrated system with closed circuit condition, the applied voltage as the driving energy could promote the cathode reaction 
(Cui et al., 2014). AO7 as the only electron acceptor in the cathode chamber could be effectively reduced to realize the electrochemical decolorization (Mu et al., 2009). In conclusion, a small amount of azo dye was decolorized through the anaerobic sludge, and AO7 was primarily decolorized through the electrochemical reaction at cathode in the integrated system.

Further considering that the sludge could realize the anaerobic decolorization and likely contribute partly to the further degradation of products (Solís et al., 2012), cathode flow direction was optimized to be down-flow direction from electrode zone to sludge zone, compared with the up-flow direction from sludge zone to electrode zone. The experimental results indicated that an optimal flow direction could be selected to maximize the performance of integrated BES-ASR. When the reactor operated in the up-flow mode, the decolorization through the sludge zone was $15.3 \pm 1.3 \%$ at $100 \mathrm{mg} \mathrm{L}^{-1}(\mathrm{HRT}=10 \mathrm{~h})$, the corresponding decolorization through the electrode zone was $76.8 \pm 2.2 \%$, resulting in the total decolorization of $92.1 \pm 1.7 \%$. Meanwhile, when the reactor was operated in the down-flow mode, the decolorization through the electrode zone was $87.1 \pm 2.6 \%$, the corresponding decolorization through the sludge zone was $9.7 \pm 1.2 \%$, resulting in the total decolorization of $96.8 \pm 2.4 \%$ (Fig. 5B). These results showed that in the down-flow mode, electrode could play a more effective role in pretreatment for about $10 \%$ higher than that in up-flow mode. This advantage could be enlarged in the higher concentrations. With the increase of AO7 concentration to $300 \mathrm{mg} \mathrm{L}^{-1}$, the down-flow mode had the ability to load a more tolerant for $90.4 \pm 3.3 \%$ decolorization compared to that of $75.5 \pm 1.1 \%$ in up-flow mode. The electrochemical decolorization by cathode was $76.2 \pm 2.2 \%$ and $70.1 \pm 1.1 \%$ for down-flow direction and up-flow direction, respectively, while the corresponding anaerobic decolorization by microorganisms was $14.2 \pm 2.4 \%$ and $5.4 \pm 1.1 \%$ (Fig. 5B). As can be seen, the down-flow direction performed better decolorization in both electrode zone and sludge zone. Considering that the azo bond cleavage through electron supply in BES can be known as a pretreatment process in azo dye decolorization (Mu et al., 2009), in the down-flow direction, the AO7 concentration might be reduced after going through the cathode for pretreatment, which made low toxic effect on the anaerobic microorganisms in the sludge and then improved the final decolorization efficiency.

Changing the flow direction to be down-flow also showed success in improving its performance on COD removal from $23.5 \pm 1.3 \%$ to $5.1 \pm 0.7 \%$ compared to that of $13.0 \pm 1.2 \%$ to $1.6 \pm 0.3 \%$ in up-flow mode as concentration increased from 100 to $300 \mathrm{mg} \mathrm{L}^{-1}$ (Fig. 5C). Take $100 \mathrm{mg} \mathrm{L}^{-1}$ for example, the COD removal in up-flow mode was $11.5 \pm 1.4 \%$ for sludge and $1.5 \pm 0.9 \%$ for cathode, and that for down-flow mode was $21.5 \pm 1.5 \%$ and $2.0 \pm 1.1 \%$, respectively (Fig. 5 C). Results showed that the effect of flow sequence on COD removal was lack of significance in the electrode zone, and the final COD removal was mainly caused by the sludge zone. It might be due to that the down-flow direction played a major role in realizing azo bond cleavage firstly through cathode as a pretreatment process (Mu et al., 2009), leading to the reduction decolorization with a little COD removal (Fig. 5B and C). Subsequently, the products of azo dye could be further treated through sludge, resulting in significant removal of COD in sludge zone (Fig. 5C). Although up-flow is widely used to treat wastewater, however, its capacity is severely confined by clogging problems, which are common during the wastewater treatment processes (González-Gutiérrez et al., 2015; Shen et al., 2014). Zhu et al. recommended that the flow direction should be considered for the proper design of BES by down-flow (Zhu et al., 2011), which was similar to this work. Especially considering the difficulty of azo bond cleavage that might lead to poor treatment performance in anaerobic sludge reactor (Solís et al., 2012), this improvement with down-flow direction in the integrated system will bear more potential to achieve the double goals of low energy consumption and advanced wastewater treatment.

\subsection{Optimization of the catholyte flow regimes between cathode and sludge}

BES mass transfer and associated over-potential limitations can be affected by flow regimes, which may simultaneously influence the electrochemical performance and pollution treatment capacities (Haeger et al., 2014; Kim et al., 2012; Liao et al., 2015; Zhang et al., 2013). Taking into account of the fully utilization of cathode in the integrated system, this study executed a new operation mode by changing the planar catholyte distributor to be a spiral one, to further improve the performance of the $1 / 4$ soaking integrated system with down-flow direction at HRT of $8 \mathrm{~h}$.

The comparison of planar distributor and spiral distributor experiments showed that the integrated system with novel spiral distributor achieved better decolorization than the traditional planar one, appeared to be a reasonable distributor for azo dye treatment. At AO7 concentration of $100 \mathrm{mg} \mathrm{L}^{-1}$, the decolorization was $95.3 \pm 1.7 \%$ and $88.4 \pm 2.6 \%$ for spiral water distributor and planar distributor, respectively. As it increased to $300 \mathrm{mg} \mathrm{L}^{-1}$, the BESASR with the spiral water distributor performed a decolorization efficiency of $79.4 \pm 2.9 \%$, about $15 \%$ higher than that with the planar shape ( $65.5 \pm 1.9 \%$ ) (Fig. $6 \mathrm{~A}$ ). This application of spiral distributor might have a good water distribution role in promoting mixing and creating a spiral flow around the cathode-microbes-s olutions interface (Michie et al., 2014), which could accelerate the transport of ions and chemicals that were key factors to decolorization (Zhang et al., 2013). Current analysis revealed that the spiral could improve the electrochemical activity of the biofilm. The current of spiral distributor and planar distributor was $0.032 \pm 0.003 \mathrm{~A}$ and $0.019 \pm 0.004 \mathrm{~A}$, respectively (Fig. 6B), demonstrating that the spiral flow with spiral distributor might give the enhanced electron transfer between cathode electrode and azo dye through promoting the azo dye distribution to electrochemically-active bacteria at cathode. It has found that the level of mixing and the associated shear rates presented in the spiral design of electrode affected both the electrical development and biofilm formation (Michie et al., 2014). The application of water flow change has also been proved to be effective in BES with spiral spacer (Zhang et al., 2013). It can maximize electro-biocatalytic activity and increase the available surface area for microbial colonization along with optimizing the mass transfer into the biofilm and protons/electrons out of the biofilm. These results supported the importance of spiral flow regime between cathode and sludge on system performance, which should be considered in optimizing the design of integrated system.

\subsection{Significances and potential}

Despite the wide range application of bioelectrochemical system and its promising perspectives, there will be numerous hurdles need to be addressed before its field application (Zhang and Angelidaki, 2014). Hence, research in this field is now focused on finding an optimal process related to different current technologies in order to achieve its advantages and to evaluate its industrial feasibility on a large scale. In this sense, it would be better to inset BES into other reactors to form an integrated system, which is cost-effective and scaling-up applicable (Kong et al., 2015; Zhang and Angelidaki, 2014). Our previous study has developed a new integrated system, embedding a modular bioelectrochemical system (BES) with surrounding electrode deployment into an anaerobic sludge reactor (ASR), to improve azo dye decolorization (Kong et al., 2015). This compact structure with large area and small 

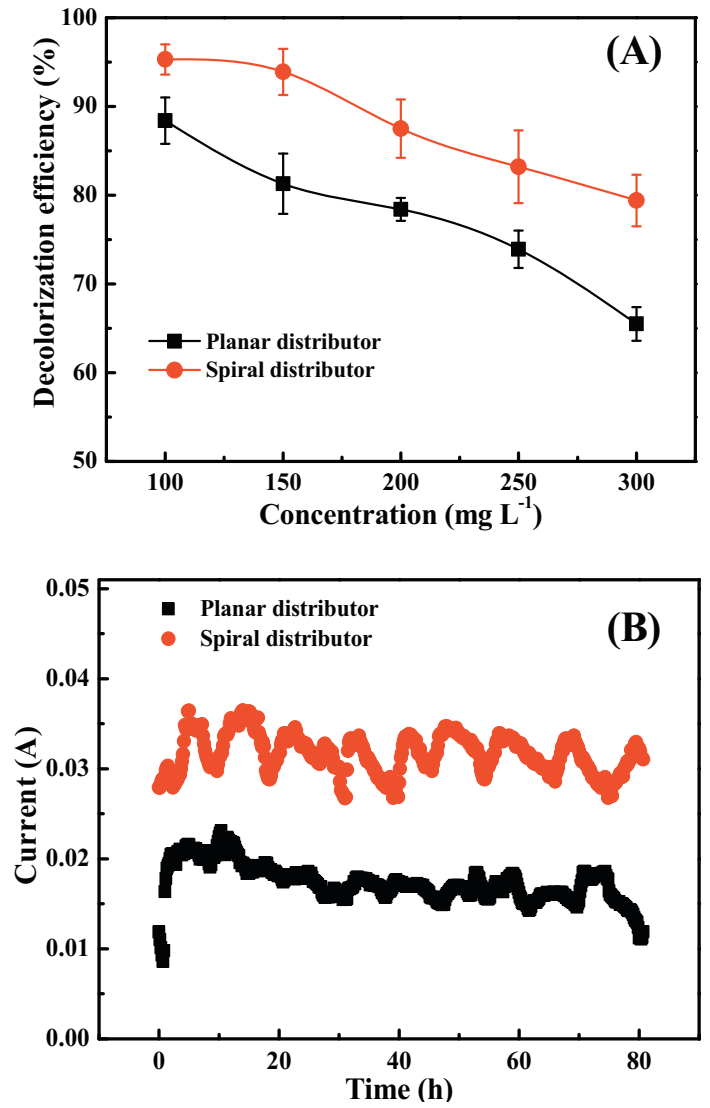

Fig. 6. The decolorization efficiency (A) and current (B) with planar catholyte distributor and spiral distributor (HRT $=8 \mathrm{~h})$.

distance between anode and cathode as well as the flexible placement of anode and cathode has proved to not only reduce the BES's internal resistance but also enhance its adaptability to wastewater (Kong et al., 2013). This kind of integrated system may offer a new sustainable approach and useful information for the efficient treatment of wastewater. However, there are still some challenges for this technology application, including the optimization of the integrated system, the interaction between the BES and anaerobic sludge, and the tolerance issues in the integrated system. Moreover, cathodic reaction determined the azo dye decolorization in the integrated system. The effective reaction at cathode could improve the stability of the reactor, increase the processing load, and reduce the charge transfer resistance.

Thus, in this study, the matching modes of BES module and anaerobic sludge reactor were discussed and optimized in the terms of relative positions, flow sequences and flow regimes between cathode electrode and sludge. These factors showed important roles in cathode reaction. The $1 / 4$ cathode soaking into sludge was suitable and had no adverse effect on the electrogenic biofilm and biocatalytic activity, likely due to the efficient proton transfer and electron transfer (Figs. 1-4). Moreover, the flow direction behavior of the integrated system was one of the key parameters that influence performance, which made it challenging to control and characterize the interaction between bioelectrochemical system and anaerobic sludge process (Fig. 5). The novel spiral water distributor was determined to be an optimum integration of matching mode (Fig. 6). The main goal of water distributor was to enhance the microbial activity through the intense interaction among pollutants, microorganisms, and cathode without significant alteration in the system operation (Michie et al., 2014). After optimizing the integrated system through these three matching modes, the biofilm will be easily and rapidly formed with $1 / 4$ cathode soaking and the azo dye could be evenly distributed inside the cathode chamber with spiral distributor surrounding the cathode, and thereby inhibiting the creation of dead zones where electrode surface area is not efficiently used for decolorization. The cathodic degradation process eliminated the antibacterial activity of azo dye at cathode zone, and promoted further COD removal in the sludge zone with the down-flow sequence from cathode zone to sludge zone. These matching modes optimization will enlarge the potential application of integrated system with bioelectrochemical system and anaerobic sludge reactor for refractory azo dye wastewater treatment.

\section{Conclusions}

Improved wastewater (azo dye) treatment was demonstrated through optimizing the matching modes of bioelectrochemical module and anaerobic sludge in the integrated system. Soaking condition can enhance the electron and proton transfer for decolorization. Flow sequence from electrode zone to sludge zone can favor the efficient use of cathode, improving decolorization and further COD removal on the cooperation of bioelectrochemical module and anaerobic sludge. Spiral water distributor could benefit to create a helical flow around the electrode-microbes-solution interface to promote the reaction. It will provide a theoretical basis for developing an integrated system with suitable matching modes in practical application.

\section{Acknowledgements}

This research was supported by the National Science Foundation for Distinguished Young Scholars (No. 51225802), the Shanghai Tongji Gao Tingyao Environmental Science \& Technology Development Foundation (STGEF), and the National High-tech R\&D Program of China (No. 2012AA051502).

\section{Appendix A. Supplementary data}

Supplementary data associated with this article can be found, in the online version, at http://dx.doi.org/10.1016/j.biortech.2015.06. 001.

\section{References}

Cui, D., Guo, Y.-Q., Lee, H.-S., Wu, W.-M., Liang, B., Wang, A.-J., Cheng, H.-Y, 2014 Enhanced decolorization of azo dye in a small pilot-scale anaerobic baffled reactor coupled with biocatalyzed electrolysis system (ABR-BES): a design suitable for scaling-up. Bioresour. Technol. 163, 254-261.

Fernando, E., Keshavarz, T. Kyazze, G., 2012. Enhanced bio-decolourisation of acid orange 7 by Shewanella oneidensis through co-metabolism in a microbial fuel cell. Int. Biodeterior. Biodegrad. 72, 1-9.

González-Gutiérrez, L. Frontana, C., Martínez, E. 2015. Upflow fixed bed bioelectrochemical reactor for wastewater treatment applications. Bioresour. Technol. 176, 292-295.

Haeger, A., Forrestal, C., Xu, P., Ren, Z.J., 2014. High performance spiral wound microbial fuel cell with hydraulic characterization. Bioresour. Technol. 174, 287-293.

He, Z., Mansfeld, F., 2009. Exploring the use of electrochemical impedance spectroscopy (EIS) in microbial fuel cell studies. Energy Environ. Sci. 2, 215-219.

Huang, L., Yao, B., Wu, D., Quan, X., 2014. Complete cobalt recovery from lithium cobalt oxide in self-driven microbial fuel cell - microbial electrolysis cell systems. J. Power Sources 259, 54-64.

Kim, J.R., Boghani, H.C., Amini, N., Aguey-Zinsou, K.-F., Michie, I., Dinsdale, R.M. Guwy, A.J., Guo, Z.X., Premier, G.C., 2012. Porous anodes with helical flow pathways in bioelectrochemical systems: the effects of fluid dynamics and operating regimes. J. Power Sources 213, 382-390.

Kong, F., Wang, A., Cheng, H., Liang, B., 2014a. Accelerated decolorization of azo dye Congo red in a combined bioanode-biocathode bioelectrochemical system with modified electrodes deployment. Bioresour. Technol. 151, 332-339.

Kong, F., Wang, A., Liang, B., Liu, W., Cheng, H., 2013. Improved azo dye decolorization in a modified sleeve-type bioelectrochemical system. Bioresour. Technol. 143, 669-673. 
Kong, F., Wang, A., Ren, H.-Y., 2014b. Improved 4-chlorophenol dechlorination at biocathode in bioelectrochemical system using optimized modular cathode design with composite stainless steel and carbon-based materials. Bioresour. Technol. 166, 252-258.

Kong, F., Wang A., Ren, H.-Y. 2015. Improved azo dye decolorization in an advanced integrated system of bioelectrochemical module with surrounding electrode deployment and anaerobic sludge reactor. Bioresour. Technol. 175, 624-628.

Kong, F., Wang, A., Ren, H.-Y., Huang, L., Xu, M., Tao, H., 2014c. Improved dechlorination and mineralization of 4-chlorophenol in a sequential biocathode-bioanode bioelectrochemical system with mixed photosynthetic bacteria. Bioresour. Technol. 158, 32-38.

Li, W.-W., Yu, H.-Q., He, Z., 2014. Towards sustainable wastewater treatment by using microbial fuel cells-centered technologies. Energy Environ. Sci. 7, 911-924.

Liao, Q., Zhang, J., Li, J., Ye, D., Zhu, X., Zhang, B., 2015. Increased performance of a tubular microbial fuel cell with a rotating carbon-brush anode. Biosens. Bioelectron. 63, 558-561.

Michie, I.S., Kim, J.R., Dinsdale, R.M., Guwy, A.J., Premier, G.C., 2014. The influence of anodic helical design on fluid flow and bioelectrochemical performance. Bioresour. Technol. 165, 13-20.

Mu, Y., Rabaey, K., Rozendal, R.A., Yuan, Z., Keller, J., 2009. Decolorization of azo dyes in bioelectrochemical systems. Environ. Sci. Technol. 43, 5137-5143.

Pant, D., Van Bogaert, G., Diels, L., Vanbroekhoven, K., 2010. A review of the substrates used in microbial fuel cells (MFCs) for sustainable energy production. Bioresour. Technol. 101, 1533-1543.
Ruiz, Y., Baeza, J.A., Guisasola, A., 2015. Enhanced performance of bioelectrochemical hydrogen production using a $\mathrm{pH}$ control strategy. ChemSusChem 8, 389-397.

Shen, J., Xu, X., Jiang, X., Hua, C., Zhang, L., Sun, X., Li, J., Mu, Y., Wang, L., 2014. Coupling of a bioelectrochemical system for p-nitrophenol removal in an upflow anaerobic sludge blanket reactor. Water Res. 67, 11-18.

Solís, M., Solís, A., Pérez, H.I., Manjarrez, N., Flores, M., 2012. Microbial decolouration of azo dyes: a review. Process Biochem. 47, 1723-1748.

Sun, J., Bi, Z., Hou, B., Cao, Y.-O., Hu, Y.-Y., 2011. Further treatment of decolorization liquid of azo dye coupled with increased power production using microbial fuel cell equipped with an aerobic biocathode. Water Res. 45, 283-291.

Tao, H.-C., Lei, T., Shi, G., Sun, X.-N., Wei, X.-Y., Zhang, L.-J., Wu, W.-M., 2014. Removal of heavy metals from fly ash leachate using combined bioelectrochemical systems and electrolysis. J. Hazard. Mater. 264, 1-7.

Wang, H., Ren, Z.J., 2014. Bioelectrochemical metal recovery from wastewater: a review. Water Res. 66, 219-232.

Zhang, F., Ge, Z., Grimaud, J., Hurst, J., He, Z., 2013. Improving electricity production in tubular microbial fuel cells through optimizing the anolyte flow with spiral spacers. Bioresour. Technol. 134, 251-256.

Zhang, Y., Angelidaki, I., 2014. Microbial electrolysis cells turning to be versatile technology: recent advances and future challenges. Water Res. 56, 11-25.

Zhu, F., Wang, W., Zhang, X., Tao, G., 2011. Electricity generation in a membraneless microbial fuel cell with down-flow feeding onto the cathode. Bioresour. Technol. 102, 7324-7328. 